\title{
COMPUTERS IN GENERAL PRACTICE-II
}

\author{
Andrew Millman, Nicholas Lee, Andrew Brooke
}

\section{Appointments and referrals}

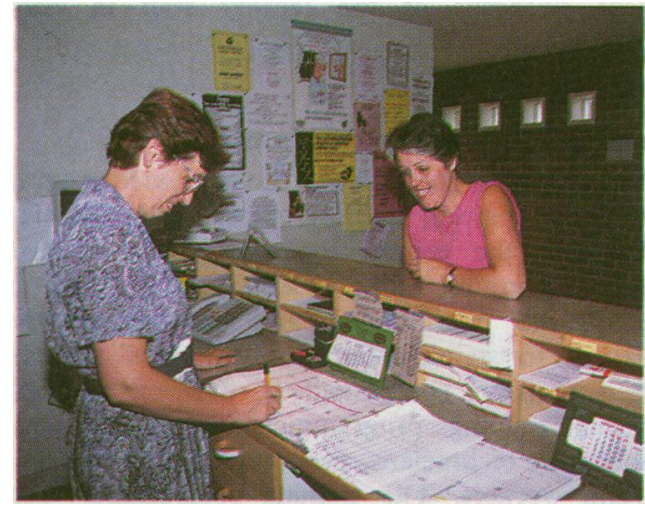

Use of traditional appointment book

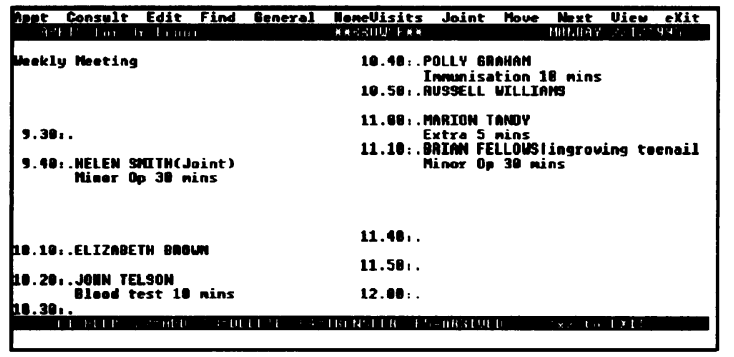

Computerised appointment system.
Computerised appointment systems offer great flexibility in making and checking appointments but have to be extremely well written to be more usable than the traditional appointment book at the reception desk. One strength of the book is that it readily allows the receptionist an overview of activity in the practice, whereas computerised systems often show just one doctor's appointments at a time, making it more difficult to spot gaps where an urgent appointment could be fitted in.

Computerised systems do, however, have several useful advantages. Good systems automatically offer patients a choice of dates and times. Access to the appointment system is available at all terminals in a practice, and it is therefore possible for a doctor or practice nurse to make a follow up appointment before a patient leaves the consulting room. Making an appointment this way can be as simple as typing $2 \mathrm{~W}$ or $3 \mathrm{M}$ (for two weeks or three months respectively). The system then searches for a vacant slot at about the right time. This relieves some of the pressure on receptionists, freeing time for other clerical tasks.

Most computerised systems automatically generate lists of patients to be seen by each doctor. During a consultation, the relevant list can be viewed on the doctor's terminal. Simply pointing to a name on the list calls up the patient's record, saving the time taken to search for it. Computerised systems also provide practices with statistics on consultation rates and workload and can be used to audit waiting times and to help practices to meet the targets set in the patient's charter.

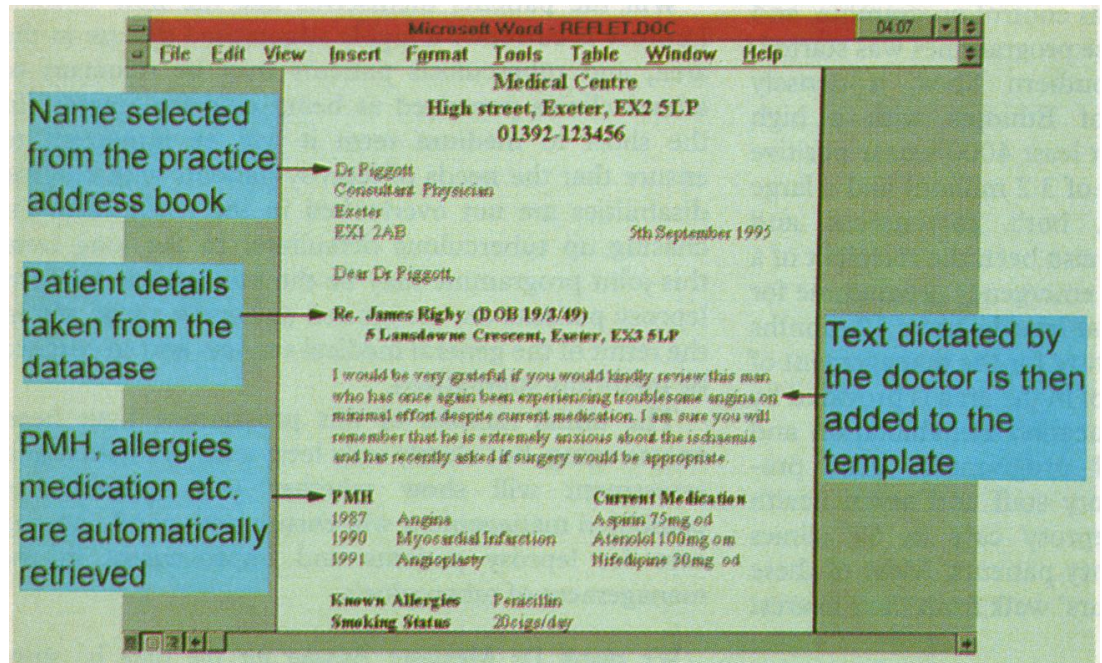

Creating a referral letter from computer database. These same details can be passed automatically to fundholding software for future reconciliation with an invoice from the hospital.
General practitioners write many referral letters each week, making a great deal of work for a practice secretary. Good letters contain details of a patient's medical history, current problems, and any drugs being taken. All of these details are held in the database and can therefore be retrieved for use in referral letters-saving the trouble of typing them out each time. Furthermore, you can create an address book containing details of the hospitals, clinics, and consultants to whom your practice's patients are commonly referred. Most systems include a basic word processor linked to the database and a series of customisable letter templates.

To create a referral letter, the secretary picks an appropriate template, selects the relevant consultant from the address book, and then merges both registration and clinical data from the patient's record into the template letter. Although this sounds complex, it can usually be done with just a few keystrokes. This saves time and reduces the risk of error in transcribing from a patient's notes. It is then a simple matter to type in the text dictated by the doctor.

At present letters are generally printed out and sent by post, but in future they are likely to be sent to their destination electronically via the NHS network. 


\section{Support}

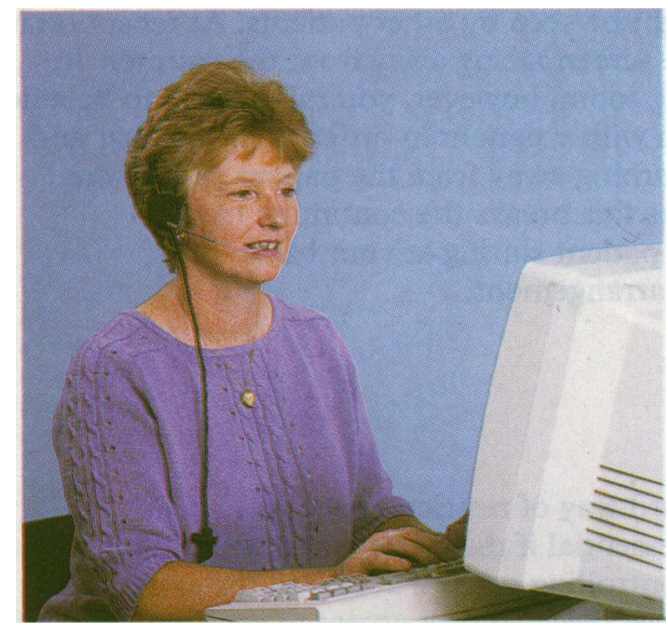

Computer help desks provide easy access to expert advice.

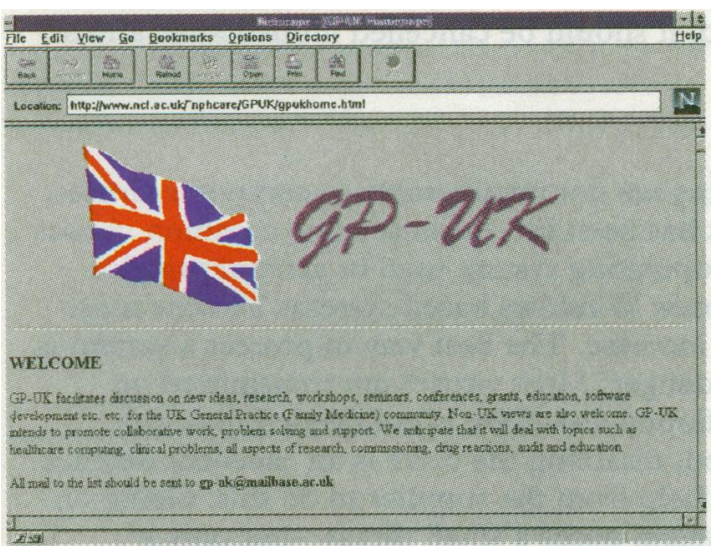

GP-UK Internet home page.

\section{Service contracts}

Computer systems are generally very reliable, but both hardware and software sometimes fail. Practices that are dependent on a computer to perform key tasks are vulnerable to considerable disruption if the system crashes. It is therefore essential that the practice has a maintenance contract with the supplier of the system and easy access to a help desk during normal surgery hours. Many crashes are not due to equipment failure but occur because of operator error. Fortunately, many of these problems are relatively simple to sort out in a few minutes given clear advice over the telephone. More complex problems are best resolved by the supplier using a modem and remote control software. This allows the supplier to take control of the system to fix the problem, after which it is returned to your control.

Hardware failures are more of a problem, and it is usually necessary to call out an engineer to repair the fault. It is sometimes possible to avoid call outs and reduce maintenance costs by keeping a few simple spares in stock. Dumb terminals, for example, can be replaced easily without special knowledge. The maintenance contract should stipulate the maximum call out time-preferably no more than eight hours. Before committing to a particular computer system, practices should consult existing users to ascertain the effectiveness of thè support offered to them in past crises.

\section{User groups}

In addition to support from the supplier, colleagues who have long experience of using the system are often able to answer common questions and may well have found a way around apparent limitations in the software. It is therefore worthwhile finding out if there is a user group in your area. Most run regular, well supported meetings. In addition to problem solving and disseminating information about future developments, these groups provide valuable feedback to suppliers. Information gained from users can be used to track down and resolve bugs in the software and stimulates development of new features in response to popular demand.

Some user groups run bulletin board systems (BBS), allowing anyone to dial in, read the latest news, post messages and questions, and retrieve answers. The national bulletin boards provide a means of making contact with distant colleagues who share similar interests and are well worth exploring. The Internet opens up new possibilities. The Primary Healthcare Specialist Group of the British Computer Society and the Sowerby Unit at Newcastle University both run a series of worldwide web pages containing a wealth of information about new developments. These pages are available to primary care physicians anywhere in the world.

Training goes hand in hand with support. It is essential to set aside time for effective training in order to maximise the return on your investment. Suppliers of new systems are usually keen to provide training as soon as possible after installation as this reduces the workload on their help desk. It is most cost effective for key members of staff to receive this expert training first. When they have mastered the system their skills can be cascaded through a practice at little expense. New staff merit particular attention, and everyone should have a periodic update as computer systems tend to evolve slowly. Failure to address this important issue leaves a practice vulnerable to errors and financial loss.

Training session. 


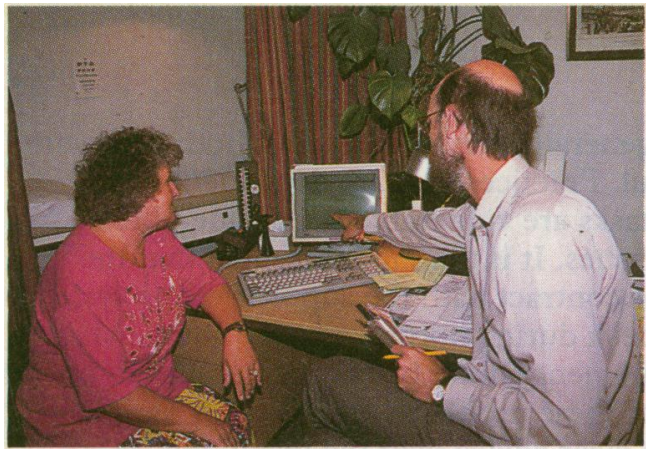

Using computer in consulting room.

\section{Security}

\section{Choosing a password}

- Use minimum of six characters

- Avoid obvious names (such as those of children or pets)

- Avoid complex combinations (such as "GTFAZROS")

- Consider using two common but unconnected words separated by a typographic symbol: "tree/dog," "grass@chair," or "money\#water"

- Never share your password

- Change your password regularly

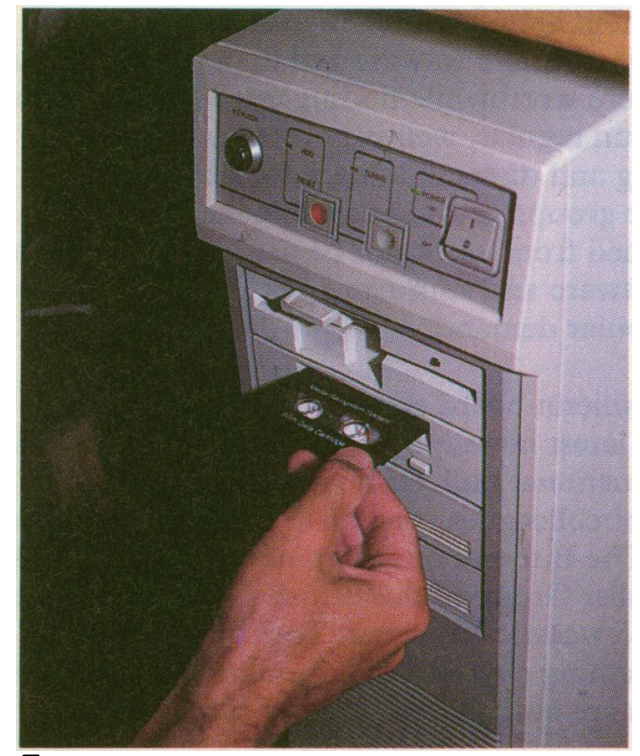

Tape streamer.

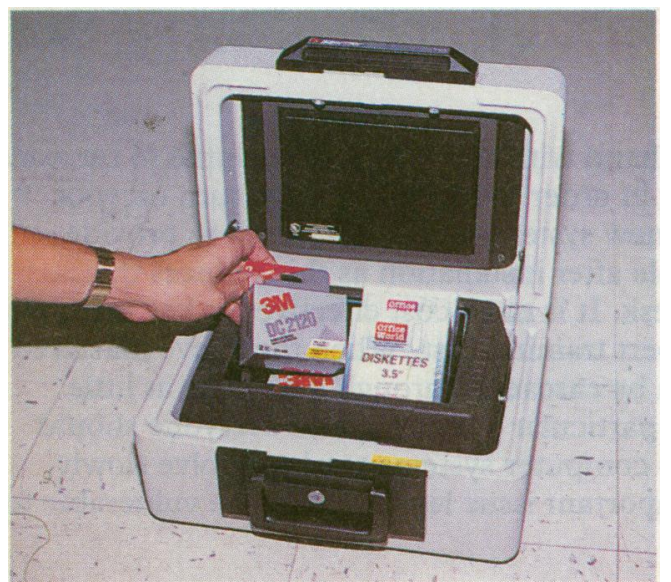

Fireproof data safe.

\section{Positioning the computer screen}

It is also worth devoting some time to other important factors such as the way people interact with a computer while dealing with a patient. Staff should be taught to be careful not to leave confidential data on screen where it might be seen by other patients. At reception it is therefore best to have the screen facing away from the counter. In the privacy of the consulting room, however, you might wish to be able share information on screen with a patient in order to foster trust and to reinforce your advice. Turning away from the patient to face the computer is far from ideal as this breaks the continuity of the consultation and leaves the patient staring at your back. The photograph shows an ideal arrangement.

\section{Passwords}

Passwords are the standard way of restricting access to most computer systems and are essential if the system is to meet official standards. In addition to controlling general access to the system, passwords can be used to selectively restrict access to sensitive parts of the database (such as staff records) and to limit users' options. Thus, receptionists can be authorised to issue repeat prescriptions but prevented from creating new ones. Furthermore, there are certain maintenance functions which, if used incorrectly, could be very damaging. Access to these should be limited to the system supervisor (usually the practice manager or the partner with responsibility for the computer system), who will have had special training to use them properly. Every member of staff is allocated a unique password by the system manager. These should never be shared or written down. If a password is compromised, it should be cancelled immediately and a new one issued.

\section{Viruses}

General practice systems are not invulnerable to computer viruses, but until recently the risk has been low because most common viruses do not infect the kind of operating system used in general practice computers. However, as new Windows based systems become more widely used, the risk will increase. The best way to protect a system is by educating staff to the danger. Most viruses are transmitted on floppy disks, and, as the floppy disk drive is not needed once the software has been installed, disabling the drive is an excellent defence. You may, however, need help from the supplier to do this. Going on line to a bulletin board system poses a small threat-but only if programs are downloaded and run before being checked with a virus scanner.

\section{Backups}

The information held on a practice computer is the most valuable part of the system and is often irreplaceable. When faults occur the database sometimes becomes corrupted, and it is impossible to get the system up and running reliably. It is therefore vitally important that a practice strictly follows a regular backup routine so that a recent copy is always available in the event of an emergency. Once any faults have been repaired, the supplier will restore data back onto the system from the most recent backup. Most current systems use a tape streamer to transfer data from the hard disk onto a tape cartridge. You should buy several tapes, regularly rotate them, and replace them annually as they tend to wear out. Backups should be taken at least once a day-more often if the system is used heavily. Some systems require that all users $\log$ off before running the backup. This can be disruptive, but it may be possible to automate the process so that it runs during a quiet period-for example, at lunchtime or overnight. You should also maintain a written log of every backup.

Never store backup tapes beside the computer as they would then be just as vulnerable to fire or theft as the computer. The best policy is to store most of the tapes in a fireproof data safe, but at least one copy should always be kept in a secure place somewhere off the premises. 


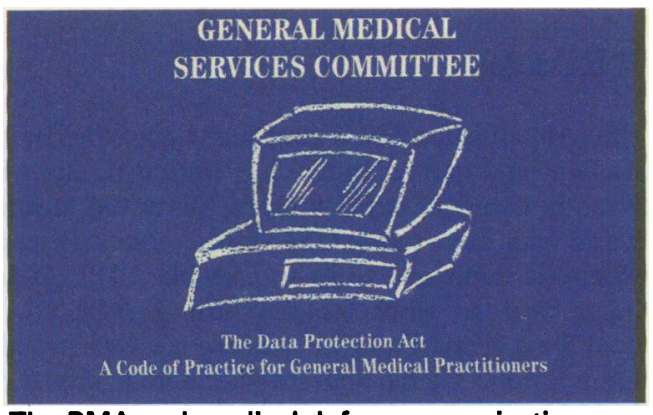

The BMA and medical defence organisations provide useful booklets on medicolegal issues.
In addition to the statutory requirement to comply with the Data Protection Act and the Display Screen Equipment Regulations, there are several specific medicolegal issues to consider when using a computer system. The current terms of service stipulate that general practitioners must record details of every consultation and prescription on paper as well as on the computer. This duplication of records is inefficient, and the government is currently looking at ways to legitimise the electronic record. Development of secure audit trails, which create an indelible chronological log of each action, will greatly ease many concerns and will be an important step along the road towards the paperless practice. At present, the medical defence organisations prefer paper records as the validity of electronic records has not yet been fully tested in the courts.

\section{Requirements for accreditation (RFA)}

The Department of Health has stipulated that, in order to qualify for the reimbursement scheme, computer systems must offer the following features (among others)

- Use of the new NHS number

- Recording of certain key details: Clinical information

Call and recall dates Item of service claims

- Read codes

- A secure audit trail

- Secure access control (passwords)

- Telephone support by supplier

- Electronic Data Interchange (EDI)

- Links to local family health services authority (registration and item of service)

- ESCROW agreement

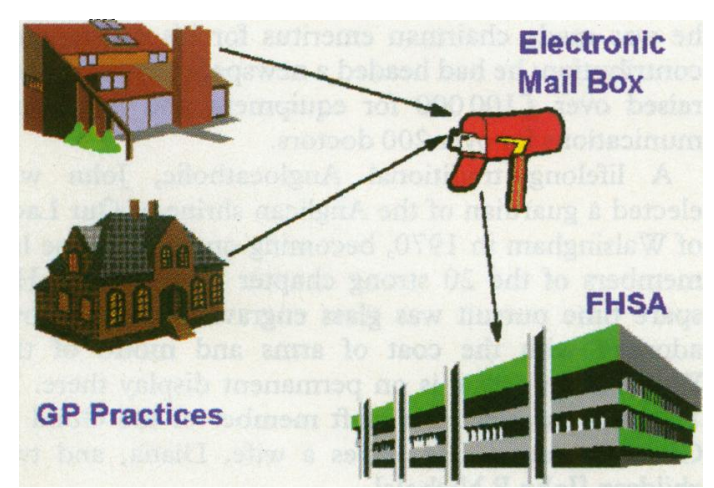

General practice links with local family health services authority.

\section{Requirements for accreditation}

The Department of Health has developed a set of minimum standards which general practice computing systems must meet if they are to qualify for reimbursement. It is likely that some suppliers will be unable to make the necessary changes to their systems and that this will result in mergers and therefore a smaller number of larger suppliers. One of the most important requirements is the establishment of an ESCROW agreement, in which the source code for the software is deposited with a third party on the understanding that it will be released to users of the system if the supplier goes out of business or withdraws from the market. This agreement is essential in order to protect users' investment in the system, which would otherwise be lost.

EDIFACT (Electronic Data Interchange For Administration Commerce and Transport) is a European standard which has been adopted by the NHS. Once it has been incorporated into all health care computer systems it will allow data to be seamlessly transferred between them. It already underpins general practices' links to family health services authorities and hospital pathology systems and is likely to form the basis for the new NHS-wide network. The links project offers the opportunity for practices to exchange data electronically with their local family health services authority. The practice computer and that of the family health services authority are not directly linked together; rather information is left in an electronic mailbox to be collected by the other computer at a convenient time. This makes the practice computer secure against unauthorised use. Initially the system is limited to the transfer of registration data-details about patients joining or leaving a practice. Later, item of service (IOS) claims may be submitted electronically, enabling a practice to claim for work done without having to complete a separate claim form for each procedure. The system is policed by a system of retrospective audit. Practices may also link up with hospital laboratories to collect results of tests the moment they have been completed. 